\title{
Relationship between magnetic resonance imaging lesions and knee symptoms in early knee osteoarthritis: A cross-sectional Japanese population study
}

\section{Seiya Ota ( $\nabla$ otaseiya012@gmail.com )}

Hirosaki university graduate school of medicine https://orcid.org/0000-0003-3988-5240

\section{Eiji Sasaki}

Department of Orthopedic Surgery, Hirosaki University Graduate School of Medicine

\section{Shizuka Sasaki}

Department of Orthopedic Surgery, Hirosaki University Graduate School of Medicine

\section{Daisuke Chiba}

Department of Orthopedic Surgery, Hirosaki University Graduate School of Medicine

\section{Yuka Kimura}

Department of Orthopedic Surgery, Hirosaki University Graduate School of Medicine

\section{Yuji Yamamoto}

Department of Orthopedic Surgery, Hirosaki University Graduate School of Medicine

\section{Mika Kumagai}

Department of Active Life Promotion, Hirosaki University Graduate School of Medicine

\section{Masataka Ando}

Department of Diet and Health Sciences, Hirosaki University Graduate School of Medicine

\section{Eiichi Tsuda}

Department of Rehabilitation Medicine, Hirosaki University Graduate School of Medicine

\section{Yasuyuki Ishibashi}

Department of Orthopedic Surgery, Hirosaki University Graduate School of Medicine

\section{Research article}

Keywords: bone marrow lesions, early knee osteoarthritis, magnetic resonance imaging, meniscal lesions, synovitis

Posted Date: July 20th, 2020

DOI: https://doi.org/10.21203/rs.3.rs-44187/v1 
License: (c) (i) This work is licensed under a Creative Commons Attribution 4.0 International License. Read Full License 


\section{Abstract}

Background: The prevalence of minute structural abnormalities in early knee osteoarthritis (KOA) and their association with knee symptoms remains unclear. We investigated the prevalence of abnormalities detected by magnetic resonance imaging (MRI) and their relation to knee symptoms in women without radiographic evidence of KOA.

Methods: This cross-sectional cohort study in a Japanese population included 261 women without radiographic evidence of KOA, which was defined as a Kellgren-Lawrence grade $<2$. All participants underwent T2-weighted fat-suppressed MRI of their right knee. Structural abnormalities (cartilage damage, bone marrow lesions [BMLs], subchondral cysts, bone attrition, osteophytes, meniscal lesions, and synovitis) were scored according to the Whole-Organ MRI Scoring method. Knee symptoms were evaluated by the Knee injury and Osteoarthritis and Outcome Score (KOOS). Participants were divided into early KOA and non-KOA groups based on early KOA classification criteria. Logistic regression analysis was performed to evaluate the relationship between MRI abnormalities and knee symptoms.

Results: Cartilage damage were the most common abnormality (53\%), followed by osteophytes (43\%), and BMLs (33\%). The prevalence of BMLs (47\%), meniscal lesions (26\%), and synovitis (37\%) was higher in early KOA than in non-KOA. On logistic regression analysis, synovitis (odds ratio [OR] $=1.901, \mathrm{P}=$ $0.013)$ and meniscal lesions $(O R=1.410, P=0.045)$ were positively associated with the presence of early KOA.

Conclusions: The prevalence of BMLs, meniscal lesions, and synovitis was higher in symptomatic knees. Synovitis was most strongly associated with knee pain and might be a therapeutic target in patients with early KOA.

\section{Background}

Knee osteoarthritis $(K O A)$ is a major public health problem in the middle-aged to elderly population and negatively affects patients' activities of daily living $(A D L)$ and quality of life $(Q O L)^{1-3}$. Early diagnosis and treatment of KOA are required to reduce its health care burden ${ }^{4}$, by preventing disease development and progression ${ }^{5-8}$. The concept of early KOA is characterized by the presence of knee symptoms in the absence of definitive radiographic abnormalities (Kellgren-Lawrence $[K L]$ grade $0-1$ ). Adults with KOA are more likely to report more knee symptoms the year before they develop radiographic evidence of $\mathrm{KOA}^{9,10}$. On the basis of this evidence, patients with early KOA may need to be targeted for therapeutic interventions as early as possible.

Pathologic changes found in osteoarthritic joints include degradation of the articular cartilage, thickening of the subchondral bone, osteophyte formation, varying degrees of synovial inflammation, degeneration of ligaments and menisci, and hypertrophy of the knee joint capsule ${ }^{11}$. Although radiographic evaluation could not detect minute structural abnormalities, such as cartilage degeneration, meniscal lesions, 
synovitis, bone marrow lesions (BMLs), and subchondral cysts, magnetic resonance imaging (MRI) may allow detection of these pathological joint tissue changes. In particular, along with knee symptoms, cartilage damage, meniscal lesions, and BMLs detected by MRI are included in the diagnostic criteria of early $\mathrm{KOA}^{5,12}$.

However, data on the structural abnormalities present in knees without radiographic changes, and on which structural changes are associated with knee symptoms, are scarce. Therefore, the present study aimed to examine the cross-sectional association between MRI lesions and knee symptoms in middleaged women without radiographic features of KOA. We hypothesized that the presence of cartilage damage, meniscal lesions, and BMLs are associated with knee symptoms, in accordance with the diagnostic criteria of early KOA.

\section{Methods}

\section{Participants}

The participants had previously volunteered for the Iwaki Health Promotion Project, which is a community-based preventative medicine program that aims to improve the average life expectancy by conducting general health checkups and prophylactic interventions, as previously described ${ }^{13-16}$. The ethics committee of the Hirosaki University Graduate School of Medicine approved the study (reference number: 2017-026), and all subjects gave written informed consent before participation.

In total, 1073 volunteers (441 men and 632 women) enrolled in the project in 2017. The participants answered questionnaires about their medical history and current health, lifestyle, and disease-specific information, such as knee symptoms. Height and weight were measured and recorded by project team staff, and body mass index (BMI) was calculated. In this study, we focused on women without radiographic abnormalities because the prevalence of KOA is higher in women ${ }^{1,17}$. Therefore, the 441 men, as well as 166 women who had radiographic features of KOA in either knee, 4 women who had undergone knee arthroplasty, 19 women who did not undergo radiographic evaluation, 176 women who lacked knee MR images, or had metal artifacts, and 6 women with a history of rheumatoid arthritis or meniscus injury were excluded. Finally, 261 women without radiographic abnormalities were included in the current analysis.

\section{Radiographic evaluation}

The presence of KOA was evaluated by weight-bearing and anterior-posterior radiographs of both knees. The beam was positioned parallel to the floor, with no angle, and aimed at the joint space. The severity of $\mathrm{KOA}$ was classified according to the $\mathrm{KL}$ classification ${ }^{18}$. A diagnosis of KOA was defined by $\mathrm{KL}$ grade $\geq 2$ in the most affected knee. All joints were graded by 2 orthopedic surgeons (DC and ES), and any discrepancy was resolved by mutual consultation. As mentioned above, only subjects who had no radiographic evidence of KOA were included in this analysis. 


\section{Knee symptoms}

Knee symptoms were scored using a patient-based outcome score, the Knee Injury and Osteoarthritis Outcome Score (KOOS). All participants completed the KOOS questionnaire independently. The KOOS questionnaire is a 42-item, knee-specific, self-administered questionnaire with 5 subscales: pain, symptoms, ADL, sports and recreation (sports), and knee-related QOL. All items were scored from 0 to 4 and the scores were then summed. Next, the raw scores were transformed to a 0-100 scale, with 100 representing the best result and 0 representing the worst ${ }^{19,20}$. Participants were divided into early KOA and non-KOA groups on the basis of their KOOS: a knee was defined as symptomatic when the scores for 2 of the 4 KOOS subscales (except KOOS sports) were "positive" (below 85\%), according to the established classification criteria for diagnosing early $\mathrm{KOA}^{6}$. Additionally, participants with joint-line tenderness or crepitation of the knee according to the symptomatic knee were classified as early KOA. Furthermore, in this study, in order to determine the association between MRI findings and knee pain, a score from 1 to 4 in each KOOS Pain item was defined as "positive", and a score of 0 was defined as "negative".

\section{Knee MRI}

All participants underwent MRI of the right knee using a rapid extremity coil and mobile MR unit (1.5 T; ECHELON RX, Hitachi, Tokyo, Japan) within 1 week of other examinations. The participants were positioned supine with their knees in full extension. Sequences included sagittal and coronal T2-weighted fat saturation fast spin echo (repetition time $5000 \mathrm{~ms}$; echo time $80 \mathrm{~ms}$; field-of-view $16 \mathrm{~cm} ; 288 \times 288$ matrix; 3-mm slice thickness, with between-slices gaps of $1.0 \mathrm{~mm}$ ). Evaluation of MRI scans was performed by 2 independent observers (DC and ES) using the Whole-Organ MRI Scoring (WORMS) method ${ }^{21}$ in a blinded fashion, with no access to the participants' clinical information. They recorded the presence of abnormalities, such as cartilage damage, bone marrow lesion, subchondral cysts, bone attrition, osteophytes, meniscal lesions, and synovitis in each subregion. In the WORMS system, the medial and lateral compartments of the tibia and femur were divided into 3 subregions (anterior, central, and posterior), and the tibia had 1 additional subregion, representing the area beneath the tibial spine. The patella was divided into medial and lateral subregions. Cartilage damage was scored in each of the 14 articular surfaces (excluding region S) from 0 (normal thickness and signal) to 6 (diffuse $[\geq 75 \%$ of the region] full-thickness loss). BMLs were each scored as integers from 0 to 3 , where $0=$ normal; $1=$ mild, $<25 \%$ of the region; $2=$ moderate, $25-50 \%$ of the region; and $3=$ severe, $>50 \%$ of the region. Subchondral cysts were graded in each region from 0 to 3 , based on the extent of regional involvement, as for BMLs. Bone attrition was defined as flattening or depression of the articular surfaces and was graded from 0 to 3 based on the subjective degree of deviation from the normal contour: $0=$ normal; $1=$ mild; $2=$ moderate; 3 = severe. Osteophytes along 14 different margins (excluding region $S$ ) of the knee were graded from 0 (none) to 7 (very large). Meniscal lesions were graded separately from 0 to $4: 0=$ intact; $1=$ minor radial tear or parrot-beak tear; 2 = non- displaced tear or prior surgical repair; $3=$ displaced tear or partial resection; 4 = complete maceration/destruction or complete resection. Synovitis was graded collectively from 0 to 3 in terms of the estimated maximal distention of the synovial cavity: $0=$ normal; $1=$ less than 
$33 \%$ of maximum potential distention; $2=33-66 \%$ of maximum potential distention; $3=$ more than $66 \%$ of maximum potential distention. The maximal total scores of cartilage damage, BMLs, subchondral cysts, bone attrition, osteophytes, meniscal lesions, and synovitis were $84,45,45,42,98,12$, and 3 , respectively.

\section{Statistical analysis}

The mean values of continuous variables (age, body mass index [BMI], and KOOS) were compared using the Mann-Whitney $\mathrm{U}$ test. The chi-square test was used to compare the prevalence of MRI lesions between non-KOA and early KOA groups. To evaluate the relationship between knee symptoms and MRI lesions, we performed crude and adjusted logistic regression analyses, with the presence of knee symptoms as an independent variable, and age, BMI, and the total score of each MRI lesion as dependent variables. Furthermore, to evaluate the relationship between knee pains and MRI lesions, we performed multiple logistic regression analyses with the presence of knee pain as an independent variable, and age, $\mathrm{BMI}$, and each MRI lesion as dependent variables. Data analysis was conducted using SPSS version $22.0 \mathrm{~J}$ (SPSS Inc., Chicago, IL, USA) in a cross-sectional manner. A p value less than 0.05 were considered statistically significant.

\section{Results}

The mean age of participants was $54.9 \pm 9.5$ years, mean BMI was $22.0 \pm 3.1 \mathrm{~kg} / \mathrm{m}^{2}$. Sixty-two $(23.8 \%)$ participants had symptomatic knees, and 51 (19.5\%) of these met the diagnostic criteria for early KOA (Table 1). The prevalence of early KOA increased with age, which exceeded $20 \%$ in participants aged 50 years and older (Fig. 1). The mean total score of cartilage damage, BMLs, subchondral cysts, bone attrition, osteophytes, meniscal lesions, and synovitis were $1.41 \pm 2.12,0.61 \pm 1.09,0.15 \pm 0.43,0.08 \pm$ $0.29,0.77 \pm 1.22,0.31 \pm 0.88$, and $0.32 \pm 0.58$, respectively. The prevalence of BMLs, meniscal lesions, and synovitis was significantly higher among participants with early KOA than among participants with nonKOA (Table 2).

According to the logistic regression analysis, $\mathrm{BMLs}$ (odds ratio $[\mathrm{OR}]=1.329,95 \% \mathrm{Cl}: 1.036,1.705$ ), bone attrition ( $\mathrm{OR}=3.128,95 \% \mathrm{Cl}: 1.280,7.642)$, meniscal lesions $(\mathrm{OR}=1.623,95 \% \mathrm{Cl}: 1.205,2.185)$, and synovitis (OR $=2.345,95 \% \mathrm{Cl}: 1.447,3.802)$ were significantly associated with the presence of early KOA. Furthermore, an adjusted regression model showed that meniscal lesions $(\mathrm{OR}=1.410,95 \% \mathrm{Cl}: 1.008$, $1.970)$ and synovitis $(\mathrm{OR}=1.901,95 \% \mathrm{Cl}: 1.148,3.150)$ were positively correlated with presence of early KOA (Table 3).

In terms of the association between MRI lesions and knee pains with certain motions, those with early KOA suffered from pain on bending the knee, climbing up or down stairs, or when performing sitting or lying down motions (Table 4). Additionally, regression analysis showed that synovitis and meniscal lesions were positively associated with almost all KOOS pain items. On the other hand, cartilage was not significantly associated with any KOOS pain item. Knee pains on climbing up or down stairs corelated with BMLs, bone attrition, and osteophytes, in addition to meniscal injury and synovitis (Table 5). 


\section{Discussion}

In this study, we investigated the relationship between MRI findings and knee symptoms in women with early KOA. The most important findings of this study were that MRI findings in early KOA, diagnosed by the newly established criteria were proven, and that imaging evidence of various types of knee pain was demonstrated. Although MRI evaluation showed that cartilage damage (53\%) was the most common finding in women without radiographic knee abnormalities, there was no significant association between cartilage damage and knee symptoms at an early phase of the disease. On the other hand, the prevalence of BMLs $(33 \%)$, bone attrition ( $8 \%)$, meniscal lesions $(16 \%)$, and synovitis $(27 \%)$ was higher in patients with early KOA. Meniscal lesions and synovitis were positively associated with knee symptoms, and key factors for various types of knee pain. These results suggested that meniscal lesions and synovitis have the greatest impact on symptoms of patients with early KOA; these should be detected and appropriate intervention applied in order to prevent disease progression.

Few studies have investigated the prevalence of abnormalities detected by MRI specifically in a population without radiographic KOA. Guermazi et al. reported that osteophytes were the most common abnormality (74\%), followed by cartilage damage (69\%), BMLs (52\%), synovitis (37\%), and bone attrition (32\%) in participants without radiographic evidence of $\mathrm{KOA}^{22}$. Javaid et al. found that osteophytes (99\%), cartilage damage $(70 \%)$, and BMLs $(59 \%)$ were common in participants without knee symptoms and radiographic $\mathrm{KOA}^{23}$. In this study, the same tendency, i.e., that many participants had cartilage damage, osteophytes, and BMLs, was observed. However, the prevalence of each abnormality was lower than that in previous reports. This may be because the participants in this study were younger, had a lower BMI, and might be at a lower risk of KOA than in previous studies.

Many previous studies have reported an association between knee effusion and knee symptoms. Torres et al. noted that synovitis or effusion detected on MRI correlated best with knee pain measured on a visual analog scale ${ }^{24}$. Chiba et al. investigated suprapatellar effusion using quantitative measurement of ultrasonography and concluded that knee effusion was associated with the symptoms as evaluated by $\mathrm{KOOS}^{15}$. Moreover, several studies have shown that knee effusion was associated with KOA disease stage and progression ${ }^{14,25,26}$. Although there have been few studies of effusion in early KOA, Harkey et al. suggested that effusion precedes the onset of accelerated KOA and may be a prognostic biomarker ${ }^{27}$. In this study, synovitis was the most strongly associated with knee symptoms and was considered to be the most important clinical finding indicating the need for intervention, in agreement with previous reports.

Meniscal lesions and BMLs were also associated with knee symptoms and disease progression in previous studies ${ }^{28-31}$. In addition, there have been reports that meniscal lesions, BMLs, and synovitis are associated with each other ${ }^{32-34}$. However, van Oudenaarde et al. have suggested that the discriminative power of single MRI features is insufficient to be useful as predictors of $\mathrm{KOA}^{35}$. In this study, although meniscal lesions, together with BMLs, bone attrition, and synovitis, were significantly associated with the presence of knee symptoms, the adjusted regression model showed that this association was attenuated 
after adjustment for age and BMI. In the early KOA population, synovitis would contribute more to knee symptoms than meniscal lesions.

BMLs reflect bony damage, which is associated with knee symptoms in KOA patients and bone metabolism, such as bone absorption. In recent studies, the presence of BMLs has been associated with knee pain and predicted cartilage loss in patients with established $\mathrm{KO}^{36-38}$. Antiresorptive drugs (e.g., bisphosphonate) were found to reduce the size of BMLs and the risk for total knee arthroplasty in KOA patients ${ }^{39,40}$. In early KOA, lower bone mineral density and higher levels of some bone markers were associated with the presence of BMLs ${ }^{16}$. In the present study, BMLs were associated with pain in relatively loaded motions, such as bending and straightening of knees, walking, and climbing stairs. BMLs may be induced by bone fragility, resulting in early subchondral changes, such as microcracking before radiologic osteoarthritic findings become definitive, and causing pain on loading.

In many previous studies, although cartilage damage was associated with bone marrow lesions ${ }^{41,42}$, meniscal damage ${ }^{43}$, and synovitis or effusion ${ }^{44}$, cartilage damage was not always associated with the severity of knee pain ${ }^{22}$. Since articular cartilage is not innervated, the association between cartilage and pain severity may be due to other structural abnormalities associated with $\mathrm{KOA}^{45}$. In this study, cartilage damage was the most common finding, but it was not significantly associated with pain. This result suggested that minor cartilage damage detected by MRI was not associated with knee symptoms in accordance with established KOA.

This study had several limitations. First, all participants in this study were women. Although the prevalence of KOA is higher in women than in men, men may be more likely to experience knee trauma, which may affect the prevalence of cartilage damage, BMLs, and meniscal lesions. Second, laboratory data, including inflammation, cartilage/bone metabolic markers, were not evaluated. Serum hyaluronic

acid, as a marker of synovitis was assessed in many previous studies ${ }^{14,46}$. Third, synovial thickening and joint effusion were not distinguished from each other, since knee effusion was not evaluated by contrastenhanced MRI. Due to the possible complications and increased cost, we did not use contrast-enhanced MRI. Fourth, the findings of this study were based on cross-sectional data; therefore, future longitudinal studies are needed to investigate whether patients with knee effusion are likely to develop KOA.

\section{Conclusion}

Structural abnormalities detected by MRI, such as cartilage damage, osteophytes, and BMLs, were commonly present in the knees of middle-aged Japanese women with no radiographic evidence of KOA. Among MRI findings, synovitis had the most marked effect on knee pain and might be a therapeutic target in patients with early KOA.

\section{Abbreviations}

$\mathrm{ADL}$ 
activities of daily living

BMI

body mass index

BMLs

bone marrow lesions

$\mathrm{Cl}$

confidence interval

$\mathrm{KL}$

Kellgren-Lawrence classification

KOA

knee osteoarthritis

KOOS

Knee injury and Osteoarthritis Outcome Score

MRI

magnetic resonance imaging

OR

odds ratio

WORMS

Whole-Organ MRI Scoring

QOL

quality of life

\section{Declarations}

\section{Ethics approval and consent to participate:}

The Ethics Committee of the Hirosaki University Graduate School of Medicine approved the study, and all participants provided written informed consent before participation.

\section{Consent for publication:}

Consent for publication was not required as no identifying personal information is being published in this manuscript.

\section{Availability of data and material:}

The datasets used and analyzed in the current study are available from the corresponding author on reasonable request. 


\section{Competing interests:}

The authors have no conflicts of interest to declare.

\section{Funding:}

This study was supported in part by a Grant-in-Aid from the Japanese Society for the Promotion of Science (Nos. 21500676, 18K16606, 18K09091), Health Labor Sciences Research Grant, JOA-Subsidized Science Project Research from the Japanese Orthopedic Association, JST COI Grant Number JPMJCE1302, and, Grant of Japan Orthopedics and Traumatology Research Foundation (No. 421).

\section{Author contributions:}

SO, ES, and DC contributed to the conception and design of the study. SO, MK and MA contributed to the acquisition of the data. SO, ES, SS, DC, YK, and YY contributed to the analysis and/or interpretation of the data. SO and ES drafted the manuscript. YY, ET, and YI revised the manuscript critically for important intellectual content. SO, ES, SS, DC, YK, YY, MK, MA, ET, and YI approved the final version of the manuscript to be published. YI is the guarantor. The corresponding author attests that all listed authors meet the authorship criteria and that no other authors meeting the criteria have been omitted.

\section{Acknowledgments:}

We are extremely grateful to all participants in the Iwaki Health Promotion Project and to the staff of our department, who conducted interviews and collected data. We would like to thank Editage (www.editage.jp) for English language editing.

\section{References}

1. Yoshimura N, Muraki S, Oka H, Mabuchi A, En-Yo Y, Yoshida M, et al. Prevalence of knee osteoarthritis, lumbar spondylosis, and osteoporosis in Japanese men and women: The research on osteoarthritis/osteoporosis against disability study. J Bone Miner Metab. 2009;27:620-8.

2. Cross M, Smith E, Hoy D, Nolte S, Ackerman I, Fransen M, et al. The global burden of hip and knee osteoarthritis: estimates from the global burden of disease 2010 study. Ann Rheum Dis. 2014;73:1323-30.

3. Kluzek S, Sanchez-Santos MT, Leyland KM, Judge A, Spector TD, Hart D, et al. Painful knee but not hand osteoarthritis is an independent predictor of mortality over 23+நyears follow-up of a populationbased cohort of middle-aged women. Ann Rheum Dis. 2016;75:1749-56.

4. Losina E, Burbine SA, Suter LG, Hunter DJ, Solomon DH, Daigle ME, et al. Pharmacologic regimens for knee osteoarthritis prevention: can they be cost-effective? Osteoarthritis Cartilage. 2014;22:415- 
30.

5. Madry H, Kon E, Condello V, Peretti GM, Steinwachs M, Seil R, et al. Early osteoarthritis of the knee. Knee Surg Sports Traumatol Arthrosc. 2016;24:1753-62.

6. Luyten FP, Bierma-Zeinstra S, Dell'Accio F, Kraus VB, Nakata K, Sekiya I, et al. Toward classification criteria for early osteoarthritis of the knee. Semin Arthritis Rheum. 2018;47:457-63.

7. Emery CA, Whittaker JL, Mahmoudian A, Lohmander LS, Roos EM, Bennell KL, et al. Establishing outcome measures in early knee osteoarthritis. Nat Rev Rheumatol. 2019;15:438-48.

8. Sasaki E, Ota S, Chiba D, Kimura Y, Sasaki S, Yamamoto Y, et al. Early knee osteoarthritis prevalence is highest among middle-aged adult females with obesity based on new set of diagnostic criteria from a large sample cohort study in the Japanese general population. 28. Arthroscopy: Knee Surgery, Sports Traumatology; 2020. pp. 984-94.

9. Case R, Thomas E, Clarke E, Peat G. Prodromal symptoms in knee osteoarthritis: a nested casecontrol study using data from the Osteoarthritis Initiative. Osteoarthritis Cartilage. 2015;23:1083-9.

10. Driban JB, Price LL, Eaton CB, Lu B, Lo GH, Lapane KL, et al. Individuals with incident accelerated knee osteoarthritis have greater pain than those with common knee osteoarthritis progression: data from the Osteoarthritis Initiative. Clin Rheumatol. 2016;35:1565-71.

11. Loeser RF, Goldring SR, Scanzello CR, Goldring MB. Osteoarthritis: a disease of the joint as an organ. Arthritis Rheumatology. 2012;64:1697-707.

12. Luyten FP, Denti M, Filardo G, Kon E, Engebretsen L. Definition and classification of early osteoarthritis of the knee. 20. Sports Traumatology: Knee Surgery; 2012. pp. 401-6.

13. Inoue R, Ishibashi Y, Tsuda E, Yamamoto Y, Matsuzaka M, Takahashi I, et al. Knee osteoarthritis, knee joint pain and aging in relation to increasing serum hyaluronan level in the Japanese population. Osteoarthritis Cartilage. 2011;19:51-7.

14. Sasaki E, Tsuda E, Yamamoto Y, Maeda S, Inoue R, Chiba D, et al. Serum hyaluronic acid concentration predicts the progression of joint space narrowing in normal knees and established knee osteoarthritis-A five-year prospective cohort study. Arthritis Research Therapy. 2015;17:283.

15. Chiba D, Tsuda E, Maeda S, Sasaki E, Takahashi I, Nakaji S, et al. Evaluation of a quantitative measurement of suprapatellar effusion by ultrasonography and its association with symptoms of radiographic knee osteoarthritis: A cross-sectional observational study. Arthritis Research Therapy. 2016;18:181.

16. Ota S, Chiba D, Sasaki E, Kumagai G, Yamamoto Y, Nakaji S, et al. Symptomatic bone marrow lesions induced by reduced bone mineral density in middle-aged women: a cross-sectional Japanese population study. Arthritis Research Therapy. 2019;21:113.

17. Felson DT, Naimark A, Anderson J, Kazis L, Castelli W, Meenan RF. The prevalence of knee osteoarthritis in the elderly. The Framingham Osteoarthritis Study Arthritis Rheumatology. 1987;30:914-8.

18. Kellgren JH, Lawrence JS. Radiological assessment of osteo-arthrosis. Ann Rheum Dis. 1957;16:494-502. 
19. Roos EM, Roos HP, Lohmander LS, Ekdahl C, Beynnon BD. Knee Injury and Osteoarthritis Outcome Score (KOOS)-Development of a self-administered outcome measure. Journal of Orthopaedic Sports Physical Therapy. 1998;28:88-96.

20. Nakamura N, Takeuchi R, Sawaguchi T, Ishikawa H, Saito T, Goldhahn S. Cross-cultural adaptation and validation of the Japanese Knee Injury and Osteoarthritis Outcome Score (KOOS). Journal of Orthopaedic Science. 2011;16:516-23.

21. Peterfy CG, Guermazi A, Zaim S, Tirman PF, Miaux Y, White D, et al. Whole-Organ Magnetic Resonance Imaging Score (WORMS) of the knee in osteoarthritis. Osteoarthritis Cartilage. 2004;12:177-90.

22. Guermazi A, Niu J, Hayashi D, Roemer FW, Englund M, Neogi T, et al. Prevalence of abnormalities in knees detected by MRI in adults without knee osteoarthritis: population based observational study (Framingham Osteoarthritis Study). BMJ. 2012;345:e5339.

23. Javaid MK, Lynch JA, Tolstykh I, Guermazi A, Roemer F, Aliabadi P, et al. Pre-radiographic MRI findings are associated with onset of knee symptoms: the most study. Osteoarthritis Cartilage. 2010;18:323-8.

24. Torres L, Dunlop DD, Peterfy C, Guermazi A, Prasad P, Hayes KW, et al. The relationship between specific tissue lesions and pain severity in persons with knee osteoarthritis. Osteoarthritis Cartilage. 2006;14:1033-40.

25. Ayral X, Pickering EH, Woodworth TG, Mackillop N, Dougados M. Synovitis. A potential predictive factor of structural progression of medial tibiofemoral knee osteoarthritis-Results of a 1 year longitudinal arthroscopic study in 422 patients. Osteoarthritis Cartilage. 2005;13:361-7.

26. Krasnokutsky S, Belitskaya-Lévy I, Bencardino J, Samuels J, Attur M, Regatte R, et al. Quantitative magnetic resonance imaging evidence of synovial proliferation is associated with radiographic severity of knee osteoarthritis. Arthritis Rheumatology. 2011;63:2983-91.

27. Harkey MS, Davis JE, Lu B, Price LL, Ward RJ, MacKay JW, et al. Early pre-radiographic structural pathology precedes the onset of accelerated knee osteoarthritis. BMC Musculoskelet Disord. 2019;20:241.

28. Kim HA, Kim I, Song YW, Kim DH, Niu J, Guermazi A, et al. The association between meniscal and cruciate ligament damage and knee pain in community residents. Osteoarthritis Cartilage. 2011;19:1422-8.

29. Chiba D, Maeda S, Sasaki E, Ota S, Nakaji S, Tsuda E, et al. Meniscal extrusion seen on ultrasonography affects the development of radiographic knee osteoarthritis: A 3-year prospective cohort study. Clin Rheumatol. 2017;36:2557-64.

30. Felson DT, Chaisson CE, Hill CL, Totterman SM, Gale ME, Skinner KM, et al. The association of bone marrow lesions with pain in knee osteoarthritis. Ann Intern Med. 2001;134:541-9.

31. Dore D, Martens A, Quinn S, Ding C, Winzenberg T, Zhai G, et al. Bone marrow lesions predict sitespecific cartilage defect development and volume loss: a prospective study in older adults. Arthritis Research Therapy. 2010;12:R222. 
32. Lo GH, Hunter DJ, Nevitt M, Lynch J, McAlindon TE, OAl Investigators Group. Strong association of MRI meniscal derangement and bone marrow lesions in knee osteoarthritis: data from the osteoarthritis initiative. Osteoarthritis Cartilage. 2009;17:743-7.

33. Roemer FW, Felson DT, Yang T, Niu J, Crema MD, Englund M, et al. The association between meniscal damage of the posterior horns and localized posterior synovitis detected on T1-weighted contrastenhanced MRI-The MOST study. Seminars in Arthritis Rheumatology. 2013;42:573-81.

34. Wang X, Blizzard L, Halliday A, Han W, Jin X, Cicuttini F, et al. Association between MRI-detected knee joint regional effusion-synovitis and structural changes in older adults: a cohort study. Ann Rheum Dis. 2016;75:519-25.

35. van Oudenaarde K, Jobke B, Oostveen AC, Marijnissen AC, Wolterbeek R, Wesseling J, et al. Predictive value of MRI features for development of radiographic osteoarthritis in a cohort of participants with pre-radiographic knee osteoarthritis-the CHECK study. Rheumatology. 2017;56:113-20.

36. Kothari A, Guermazi A, Chmiel JS, Dunlop D, Song J, Almagor O, et al. Within-subregion relationship between bone marrow lesions and subsequent cartilage loss in knee osteoarthritis. Arthritis Care Res. 2010;62:198-203.

37. Driban JB, Lo GH, Lee JY, Ward RJ, Miller E, Pang J, et al. Quantitative bone marrow lesion size in osteoarthritic knees correlates with cartilage damage and predicts longitudinal cartilage loss. BMC Musculoskelet Disord. 2011;12:217.

38. Driban JB, Price L, Lo GH, Pang J, Hunter DJ, Miller E, et al. Evaluation of bone marrow lesion volume as a knee osteoarthritis biomarker-Longitudinal relationships with pain and structural changes: data from the Osteoarthritis Initiative. Arthritis Research Therapy. 2013;15:R112.

39. Laslett LL, Doré DA, Quinn SJ, Boon P, Ryan E, Winzenberg TM, et al. Zoledronic acid reduces knee pain and bone marrow lesions over 1 year: a randomised controlled trial. Ann Rheum Dis. 2012;71:1322-8.

40. Fu SH, Wang CY, Yang RS, Wu FL, Hsiao FY. Bisphosphonate use and the risk of undergoing total knee arthroplasty in osteoporotic patients with osteoarthritis: A nationwide cohort study in Taiwan. Journal of Bone Joint Surgery American volume. 2017;99:938-46.

41. Roemer FW, Guermazi A, Javaid MK, Lynch JA, Niu J, Zhang Y, et al. Change in MRI-detected subchondral bone marrow lesions is associated with cartilage loss: the MOST Study. A longitudinal multicentre study of knee osteoarthritis. Ann Rheum Dis. 2009;68:1461-5.

42. Antony B, Venn A, Cicuttini F, March L, Blizzard L, Dwyer T, et al. Correlates of knee bone marrow lesions in younger adults. Arthritis Research Therapy. 2016;18:31.

43. Hunter DJ, Zhang YQ, Niu JB, Tu X, Amin S, Clancy M, et al. The association of meniscal pathologic changes with cartilage loss in symptomatic knee osteoarthritis. Arthritis Rheumatology. 2006;54:795-801.

44. Roemer FW, Zhang Y, Niu J, Lynch JA, Crema MD, Marra MD, et al. Tibiofemoral joint osteoarthritis: risk factors for MR-depicted fast cartilage loss over a 30-month period in the multicenter osteoarthritis study. Radiology. 2009;252:772-80. 
45. Hunter DJ, Guermazi A, Lo GH, Grainger AJ, Conaghan PG, Boudreau RM, et al. Evolution of semiquantitative whole joint assessment of knee OA: MOAKS (MRI Osteoarthritis Knee Score). Osteoarthritis Cartilage. 2011;19:990-1002.

46. Ishijima M, Watari T, Naito K, Kaneko H, Futami I, Yoshimura-Ishida K, et al. Relationships between biomarkers of cartilage, bone, synovial metabolism and knee pain provide insights into the origins of pain in early knee osteoarthritis. Arthritis Research Therapy. 2011;13:R22.

\section{Tables}

Due to technical limitations, the tabes are provided in the Supplementary Files section.

\section{Figures}




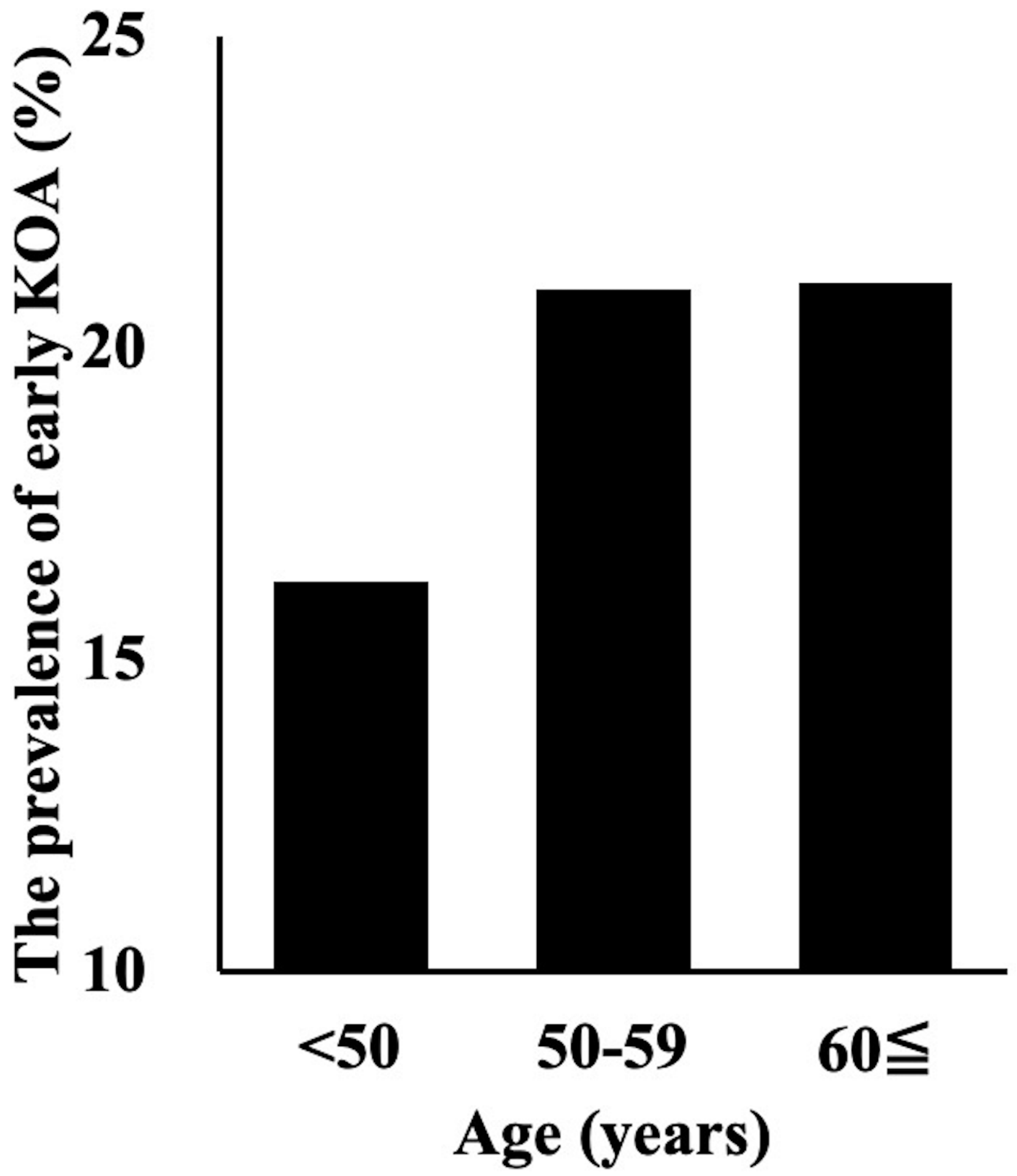

Figure 1

The prevalence of early knee osteoarthritis (KOA) classified by age

\section{Supplementary Files}

This is a list of supplementary files associated with this preprint. Click to download. 
- 200714table.docx

Page 16/16 